\title{
Diagnostic value of three-dimensional saline infusion sonohysterography in the evaluation of the uterus and uterine cavity lesions
}

\author{
Ahmed S.A. Sabry ${ }^{1 A, B, D, E, F}$, Shimaa A. Fadl2B,D,E,F, Wojciech Szmigielski ${ }^{3 A, B, D, E, F}$, Amal Alobaidely ${ }^{10, E}$, \\ Sanaa S.H. Ahmed ${ }^{1 B, D, E}$, Hanan Sherif ${ }^{4 A, B, D, E, F}$, Reda R.H.Yousef ${ }^{1 B, D, E}$, Ahmed Mahfouz ${ }^{4 A, B, D, E, F}$ \\ 'Clinical Imaging Department, Women's Wellness and Research Center, Hamad Medical Corporation, Doha, Qatar \\ 2Department of Radiology, University of Washington Medical Center, Seattle, Washington, USA \\ ${ }^{3}$ Clinical Imaging Department, National Center for Cancer Care and Research, Hamad Medical Corporation, Doha, Qatar \\ ${ }^{4}$ Clinical Imaging Department, Hamad General Hospital, Hamad Medical Corporation, Doha, Qatar
}

\section{Abstract}

Purpose: The purpose of this review is to illustrate and discuss the seldom used technique of three-dimensional (3D) saline infusion sonohysterography (SIS) based on instillation of sterile saline through a catheter into the uterus under real-time vaginal transducer observation for assessment of the endometrial cavity.

Material and methods: The cases for this pictorial review were selected from the imaging material collected at the Ultrasound Unit, Clinical Imaging Department, Women's Hospital of Hamad Medical Corporation during a sevenyear period from 2011 to 2017. This was a retrospective collection of 216 consecutive cases that underwent twodimensional (2D) and 3D SIS for evaluation of the uterine cavity, followed by verification of results with histopathology. An open-sided speculum is inserted into the vagina. An intrauterine catheter is then threaded into the endometrial cavity. After that, the speculum is removed carefully, and a vaginal transducer is inserted. Using a 60-ml syringe attached to the catheter, saline solution is instilled under direct real-time observation (2D SIS). Subsequently, reconstruction of the three-dimensional anatomy of the intrauterine cavity is performed.

Pictorial review: The review presents the most common indication for SIS, like abnormal bleeding in pre- and postmenopausal patients. SIS allows us to distinguish between focal lesions and global endometrial thickening. SIS should be supported as a second-line diagnostic procedure for abnormal uterine bleeding, when findings from transvaginal ultrasound are inconclusive.

Conclusions: The addition of 3D techniques to SIS procedure helps in the distinction between endometrial and myometrial lesions, also it facilitates delineation of uterine anatomy, resulting in more precise and accurate diagnosis.

Key words: 2 and 3 dimensions (2D and 3D), saline infusion sonography (SIS), ultrasound (US), polyps, submucous fibroids, synechiae.

\section{Introduction}

Saline infusion sonohysterography (SIS) is a minimally invasive technique in which the endometrial cavity is dis- tended with saline to allow the evaluation of a single layer of the endometrial lining. SIS enables the radiologist to reliably distinguish focal from diffuse endometrial pathologic conditions. It is less expensive, less complicated,

\footnotetext{
Correspondence address:

Wojciech Szmigielski, Clinical Imaging Department, National Center for Cancer Care and Research, Hamad Medical Corporation, P.0. Box 3050, Doha, Qatar,

e-mail:w.szmigielski@gmail.com;wszmigielski@hamad.qa
}

Authors' contribution:

A Study design - B Data collection · C Statistical analysis · D Data interpretation - E Manuscript preparation · F Literature search · G Funds collection 
and less time-consuming than hysteroscopy for detecting and characterising intracavitary abnormalities. SIS is the most commonly performed procedure to evaluate abnormal uterine bleeding in pre- and postmenopausal women. Other indications include infertility, recurrent pregnancy loss, and congenital uterine anomalies $[1,2]$.

Adding three-dimensional (3D) to conventional sonohysterography increases the accuracy of the diagnosis of endometrial pathologies. The simultaneous display of three perpendicular planes offers a more comprehensive overview of the examined area and provides access to planes unobtainable by conventional two-dimensional examinations. Surface rendering of the intracavitary pathological findings enables visualisation of their surface areas and provides valuable information about their topographical orientation [3]. This approach enables more accurate examination of the endometrial and subendometrial lesions as well as the assessment of the uterine anatomy in congenital uterine anomalies [4]. The diagnostic accuracy of this procedure is comparable to the results achieved by the diagnostic hysteroscopy [4-6].

Herein, we illustrate the technique of 3D SIS, followed by a review of $3 \mathrm{D}$ SIS characteristics of intrauterine lesions such as polyps, leiomyomas, adhesions, and endometrial hyperplasia. We describe the role of 3D SIS in the classification of congenital uterine abnormalities as well as in preoperative and postoperative cavity assessment.

The purpose of this review is to illustrate and discuss whether 3D sonohysterography (SHG) is helpful in making the important differentiation between myometrial and endometrial lesions.

\section{Material and methods}

This pictorial review is based on selected cases from the imaging material collected in the US unit, Clinical Imaging Department, Women's Hospital of Hamad Medical Corporation, during a seven-year period from 2011 to 2017. The material includes 216 patients who underwent two-dimensional (2D) and 3D SIS for evaluation of the uterine cavity followed by verification of the results with histopathology.

The age range was 18-60 years (mean age 38 years).

The examination was usually scheduled in the early follicular phase of the menstrual cycle, immediately after cessation of menstrual flow and before day 10. Day 6 is generally considered as an "ideal day" for this examination. The endometrium is relatively thin during the early proliferative phase of the cycle, which facilitates the imaging and interpretation. In the late luteal phase of the cycle, a thickened endometrium or focal irregularities in the endometrial outline may be mistaken for endometrial hyperplasia or small polyps [7]. A non-steroidal anti-inflammatory medication such as ibuprofen (400 mg i.m.) and a smooth muscle relaxant such as Buscopan (20 mg i.m.) were administered 30 minutes prior the examination as a premedication. The procedure was explained to the patient to avoid any anxiety. The patient was asked to empty their urinary bladder prior to the examination.

\section{Procedure}

The procedure was conducted in sterile conditions. The patient assumed a lithotomy position and preliminary $2 \mathrm{D}$ endovaginal scan using a 7.5-MHz mechanical probe (GE, Voluson, E8) was performed. As a baseline, unenhanced pre-instillation scanning with views of the cervix, uterus, both ovaries, and the cul-de-sac was obtained $[7,9,10]$.

A suitably sized open-sided vaginal speculum with lock (WVS1) was inserted into the vagina, and the cervix was cleansed with a povidone-iodine solution (betadine solution 10\%). Topical xylocaine gel can be used along the vaginal wall and cervix to avoid pain during the procedure [8]. An intrauterine soft balloon HSG catheter (F7) was then threaded into the endometrium [11].

Subsequently, the speculum was carefully removed without dislodging the intrauterine catheter. The tip of the catheter should lie within the cervical canal or lower uterine cavity. After that, the vaginal ultrasound transducer was re-inserted. Using a syringe, saline solution $(10-20 \mathrm{ml})$ was instilled under direct real-time observation, and a 3D volume acquisition or ultrasound sweep was performed.

Simultaneous display of the zone of interest in three perpendicular planes enhances imaging capabilities, while surface rendering provides a comprehensive overview of the imaged surface area and its topographical orientation [3] (Figure 1). The multiplane images were reviewed and, if adequate, stored on the system hard drive. Additional injection of air into the uterine cavity is possible under colour Doppler control for the visualisation of tubal patency.

It should be stressed that the image quality achieved using the $3 \mathrm{D}$ system reflects the basic $2 \mathrm{D}$ (grey-scale) per-

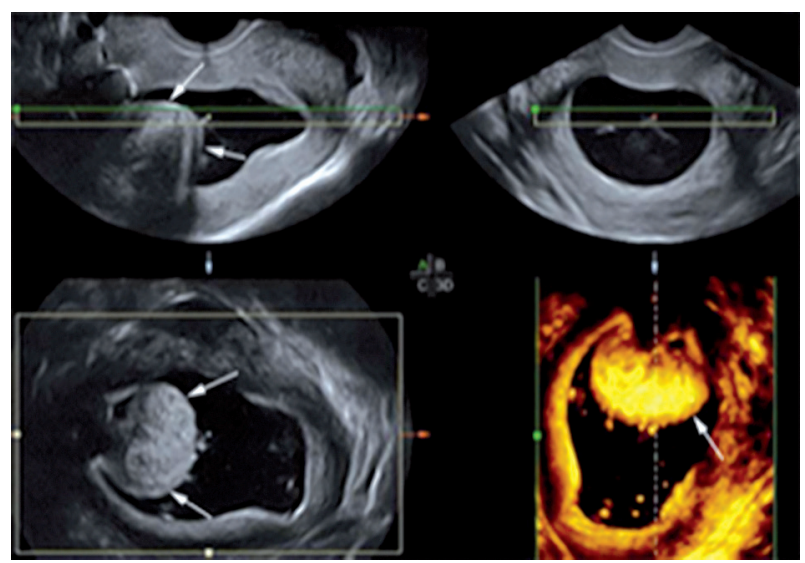

Figure 1. Submucous fibroid in a 43-year-old woman with menorrhagia. Two-dimensional saline infusion sonohysterography (SIS) demonstrates simultaneous display of the zone of interest in a submucous fibroid (arrows) in three perpendicular planes. Three-dimensional SIS (the right inferior image) enhances diagnostic imaging and topographical orientation of the submucous fibroid 


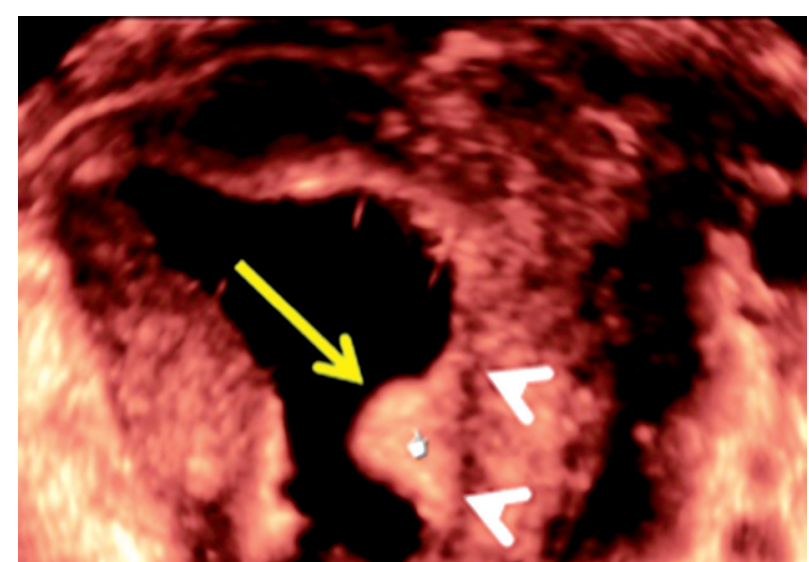

Figure 2. A sessile endometrial polyp in a 38-year-old patient with abnormal uterine bleeding. Three-dimensional saline infusion sonohysterography demonstrates a solitary, smooth, well-defined, uniformly echogenic endometrial lesion (arrow). The lesion arises from the left endometrium and has a broad-based attachment. Note the preserved endometrial/myometrial interface (arrowheads)

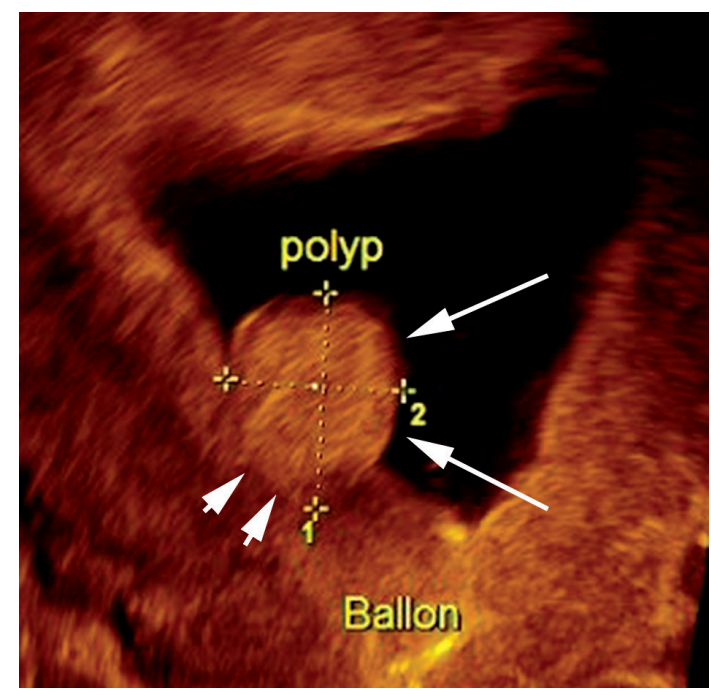

Figure 3. A pedunculated polyp in a 32-year-old woman with abnormal uterine bleeding. Three-dimensional saline infusion sonohysterography demonstrates a solitary, smooth, well-defined, uniformly echogenic endometrial lesion (arrows) arising from the right side. The polyp has a narrow-based attachment (arrowheads). Pathological analysis revealed an endometrial polyp

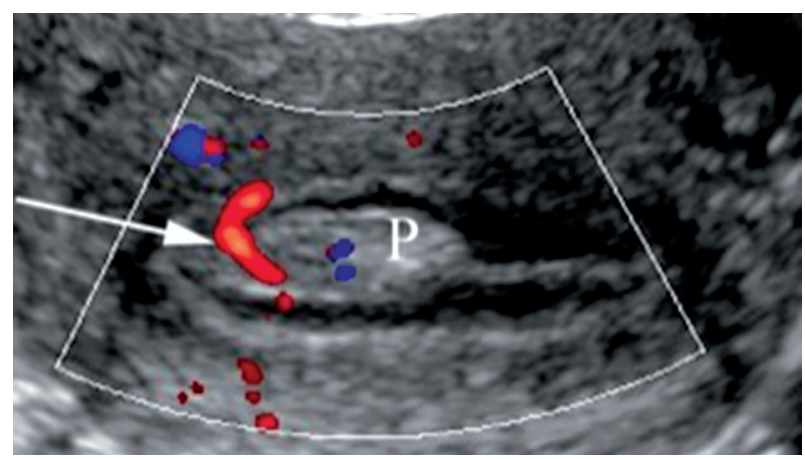

Figure 4. A pedunculated polyp in a 40-year-old woman with primary infertility. Three-dimensional saline infusion sonohysterography with volumetric reconstruction and colour Doppler demonstrates a solitary, smooth, well-defined, uniformly echogenic endometrial lesion $(P)$ arising from the anterior wall with a single feeding vessel (arrow). Pathological analysis revealed an endometrial polyp formance of the ultrasound unit with correct utilisation of both 2D and 3D controls and presets. Manipulation of the surface rendering reference plane both pre- and poststorage is of significant practical importance.

\section{Pictorial review}

This pictorial review provides examples of the usefulness of 3D SIS in the assessment of various uterine and tubal pathology.

\section{Polyps}

Polyps are localised overgrowths that may be endometrial or cervical and occur either alone or in multiples. Polyps measure from a few millimetres to centimetres and may be sessile or pedunculated. Most endometrial polyps arise from the fundal region and extend toward the internal os. Occasionally, polyps may project through the external cervical os and can be seen in the vagina [5]. The prevalence of polyps is reported to increase with age and accounts for $21 \%$ to $28 \%$ of all cases of postmenopausal bleeding $[2,5]$.

Polyps are usually observed as echogenic or isoechoic, relative to the endometrium, with preservation of the endometrial/myometrial interface [11]. Some polyps are non-uniform with small internal cysts [11]. The $3 \mathrm{D}$ reconstructed transverse and coronal planes allow accurate localisation of the point of attachment of the polyp. 3D SIS permits better assessment of the type of the polyp; polyps may be classified as sessile (Figure 2) or pedunculated (Figure 3 ) according to the ratio of the diameter of the base at the level of the endometrium ("a") and the maximal transverse diameter of the lesion ("b"). The $\mathrm{a} / \mathrm{b}$ ratio determines the type of polyp, defined as "pedunculated" if the ratio is $<1$ and "sessile" if it is 1 or more [7]. 3D SIS also is helpful in demonstrating whether there is more than one polyp. 3D SIS can demonstrate not only the point of attachment, but also it can show the presence of a single feeding vessel (stalk flow pattern) in SIS colour Doppler (Figure 4).

Differentiating endometrial polyps from submucosal fibroids can sometimes be challenging [8]; the most important criteria are listed in Table 1.

Table 1. Differences between polyps and submucosal fibroids on threedimensional saline infusion sonohysterography

\begin{tabular}{|l|l|}
\hline Polyp & Submucous fibroid \\
\hline $\begin{array}{l}\text { Mostly echogenic/isoechoic to } \\
\text { the endometrium }\end{array}$ & $\begin{array}{l}\text { Hypoechoic relative to } \\
\text { the endometrium with } \\
\text { an overlying echogenic layer }\end{array}$ \\
\hline $\begin{array}{l}\text { Single feeding vessel } \\
\text { (stalk flow pattern) }\end{array}$ & $\begin{array}{l}\text { Circular type of flow and multiple } \\
\text { feeding vessels }\end{array}$ \\
\hline $\begin{array}{l}\text { Preserved endometrial/ } \\
\text { myometrial interface }\end{array}$ & $\begin{array}{l}\text { Distorted endometrial/ } \\
\text { myometrial interface }\end{array}$ \\
\hline
\end{tabular}



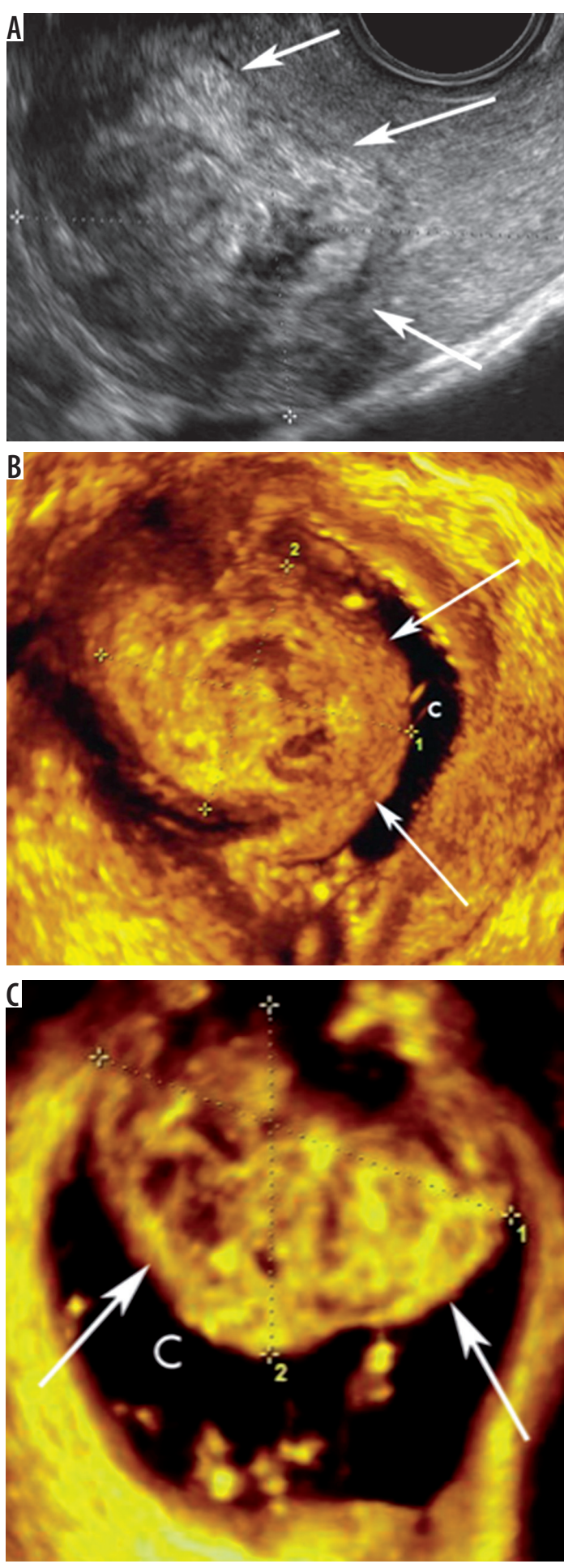

Figure 5. $\mathrm{T} 1$ submucous fibroid in a 35-year-old woman with menorrhagia. A) Two-dimensional ultrasound shows large fundal anterior heterogeneous lesion (arrows). B-C) Three-dimensional saline infusion sonohysterography reveals a broad-based fundal anterior fibroid (arrows) that protrudes into the uterine cavity $(\mathrm{C})$ by more than $50 \%$

\section{Submucous fibroids}

Submucosal leiomyomas or fibroids are a common source of abnormal uterine bleeding. They are associated with reproductive dysfunction [2] and account for approximately $10 \%$ of postmenopausal bleeding cases $[3,10]$.
In two-dimensional ultrasound (2D-US) appears intracavitary hypoechoic lesion or indenting endometrium (Figures 5A), and in sonohysterography, submucous fibroids are broad based, hypoechoic, and typically exhibit an overlying echogenic layer of endometrium (Figure 5B-C). Unlike polyps, fibroids often cause distortion to the endometrium/myometrium interface and cause acoustic shadowing [2,5]. In colour/power Doppler findings, circular flow is present [7]. Occasionally, fibroids may have a pedunculated appearance or a multilobulated surface or may be prolapsed into the endocervical canal, thereby preventing sonohysterography [2].

The anechoic interface allows the detection of the fibroid and its relationship to the endometrial cavity [12]. Submucosal fibroids often distort the endometrial cavity, with little or no intervening myometrium between the fibroid and the endometrium [12]. Intramural fibroids (Figure 6A-B) can exhibit a submucosal component that
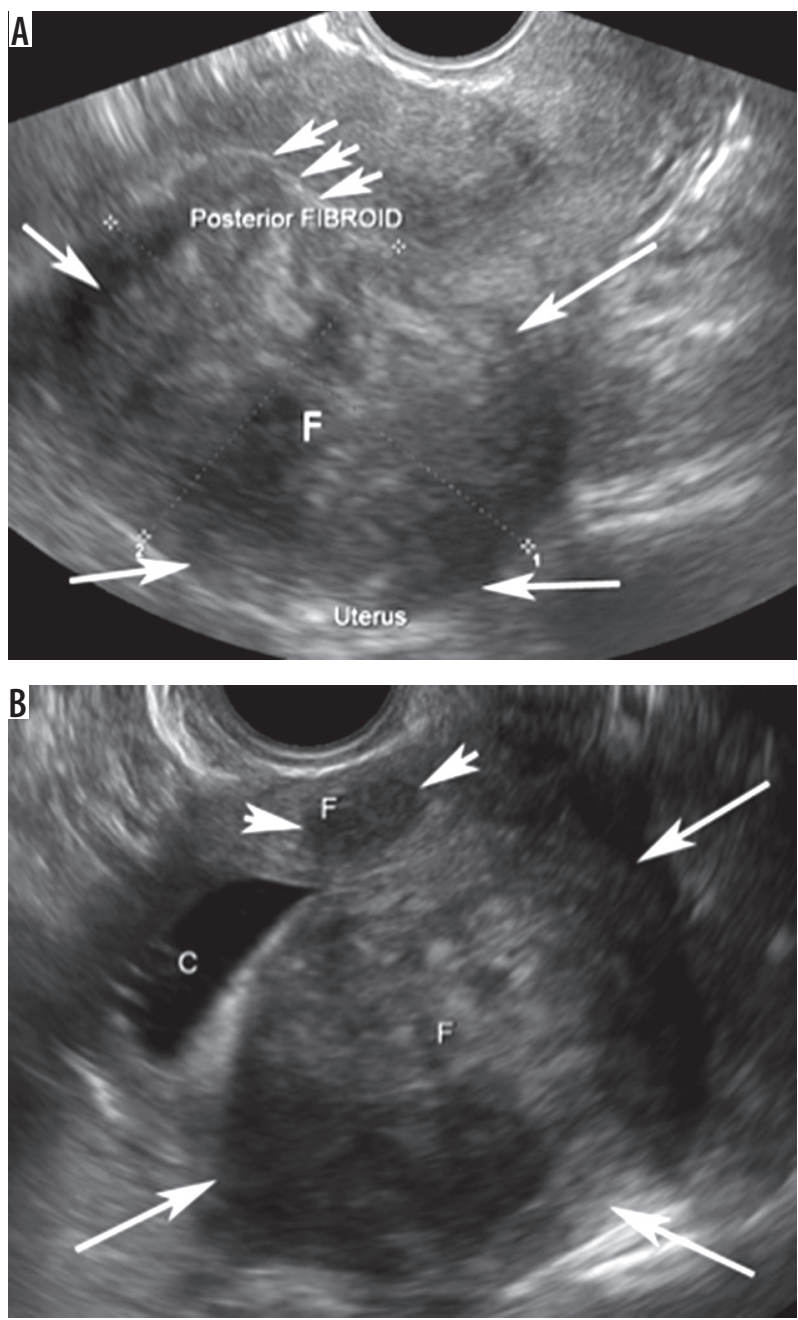

Figure 6. Intramural fibroid in a 37-year-old woman with a suspected fibroid. A) Two-dimensional ultrasound of the uterus shows a bulky uterus with posterior fibroid (arrows) deviating and indenting in the endometrium (short arrows). B) Two-dimensional saline infusion sonohysterography reveals two intramural fibroids (F). The larger one is located posteriorly (long arrows) with preserved endometrial/myometrial interface, and the smaller one (short arrows) is located anteriorly. There is deviation of the endometrial cavity 
distorts the endometrial cavity, but they are not considered submucosal fibroids unless the epicentre of the fibroid lies within the cavity [2]. Thus, by distending the inner walls of the endometrium, submucous fibroids can be differentiated from intramural fibroids that are distorting the cavity.

The major advantage of sonohysterography is that it can accurately depict the percentage of the fibroid that projects into the endometrial cavity $[2,6,10]$. Submucosal myomas can be classified into three grades according to the following criteria: (i) the widest diameter of fibroids in a plane perpendicular to the endometrium and (ii) the angle that is made between the myoma and the adjacent uterine wall. This fibroid classification (Figure 7, Table 2) can predict the success of hysteroscopic fibroid resection with a high degree of accuracy $[2,12,13]$.

3D SIS enables examination of the uterus from any angle and in any arbitrary plane. Thus, it is possible to assess both the size and the depth of myometrial extension in each individual fibroid [6]. The saved volume can be manipulated to provide measurements of the depth of myometrial extension exactly at the widest fibroid diameter, taken in a plane perpendicular to the endometrium. This approach cannot be achieved using 2D ultrasound or any other conventional technique. In addition, 3D SIS allows the assessment of complex distortions of the uterine cavity by multiple fibroids.

\section{Adenomyosis}

Adenomyosis occurs most frequently in multiparous women with presenting symptoms of abnormal uterine bleeding and dysmenorrhoea [10]. Adenomyosis is a common benign disease of the uterus characterised by ectopic endometrial glands and stroma within the myometrium associated with surrounding smooth muscle hypertrophy [14].

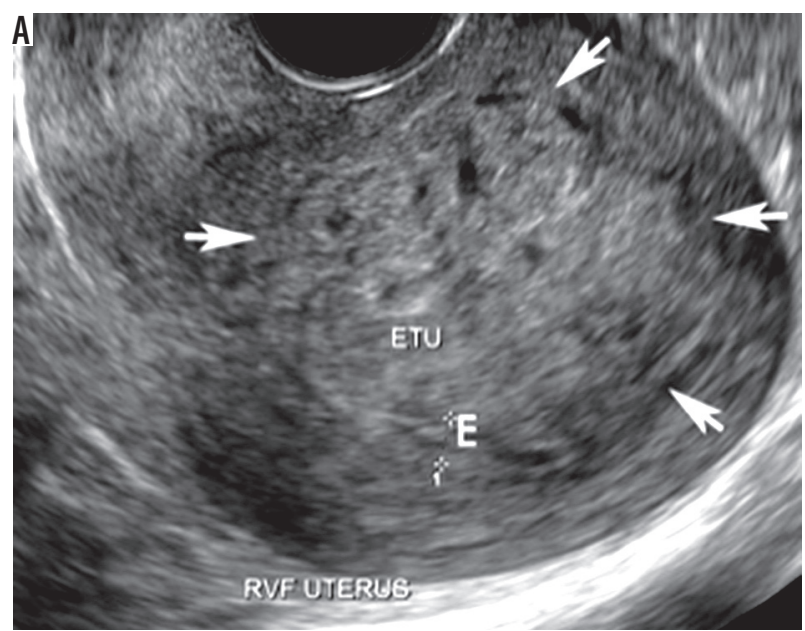

Figure 8. Focal adenomyosis in a 42-year-old woman with abnormal uterine bleeding. A) Two-dimensional ultrasound shows a retroverted uterus with thickened posterior wall (short arrows). Note the cystic changes of the thickened myometrium and poorly defined endometrium (E). B) Threedimensional saline infusion sonohysterography confirms focal asymmetric wall thickening of adenomyosis in the right posterior portion of the uterus (arrows)

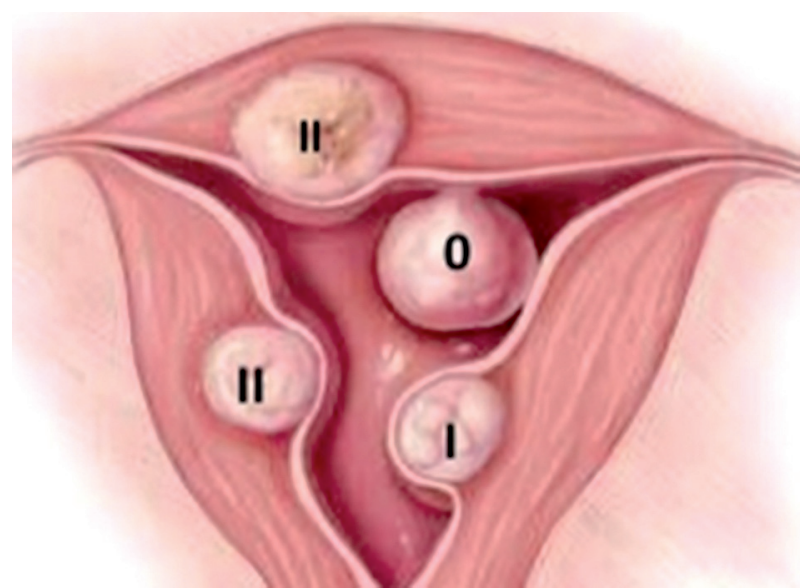

Figure 7. Grading of submucosal fibroids: T0: Fibroid totally within cavity. $\mathrm{T1}: \geq 50 \%$ in cavity. T2: $<50 \%$ in cavity (Modified from Wamsteker et al., Obstet Gynecol 1993; 82: 736-740)

Table 2. Grades of endocavitary uterine masses

\begin{tabular}{|l|l|}
\hline Grade 0 & $\begin{array}{l}\text { Complete intracavitary fibroid, pedunculated, } \\
\text { no intramural extension, angle }<20^{\circ}\end{array}$ \\
\hline Grade I & $\begin{array}{l}\text { Sessile fibroid, }>50 \% \text { of the endocavitary portion } \\
\text { protruding into the cavity, angle }<90^{\circ}\end{array}$ \\
\hline Grade II & The endocavitary portion is $<50 \%$, angle $>90^{\circ}$ \\
\hline
\end{tabular}

Sonohysterography findings include heterogeneous myometrial echotexture, asymmetric thickening of the anterior or posterior wall of the myometrium, myometrial cysts, subendometrial echogenic linear striations (myometrial cracks), and poor definition of the endometrialmyometrial junction [14] (Figure 8A).

$3 \mathrm{D}$ SIS (Figure $8 \mathrm{~B}$ ) possesses the advantage of making the important distinction between myometrial (adenomyo-

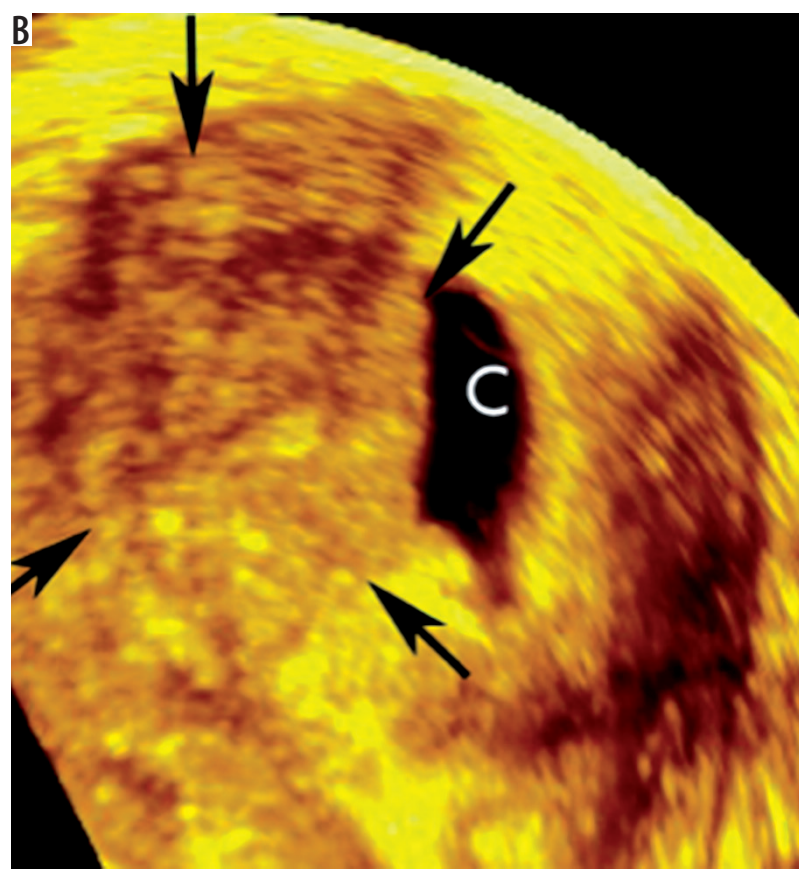




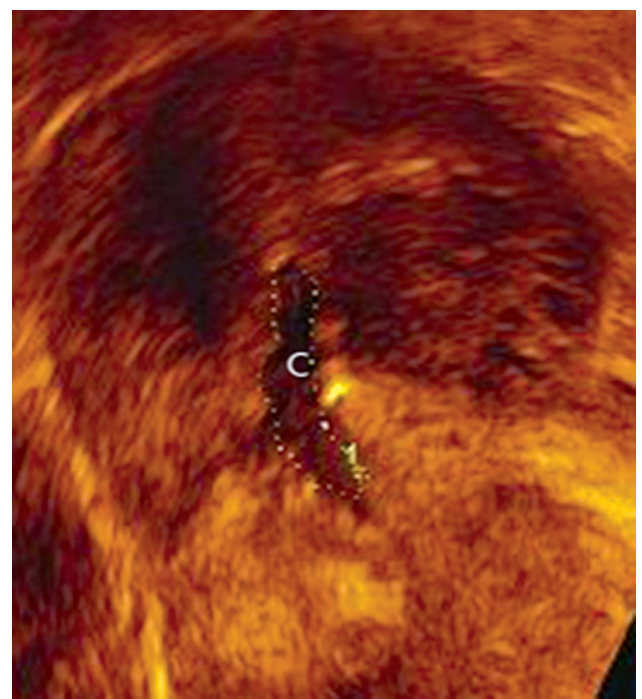

Figure 9. Asherman syndrome in a 33-year-old woman with history of secondary amenorrhea. Three-dimensional saline infusion sonohysterography shows small uterine cavity $(C)$ that is not completely distended. The uterine cavity has an irregular endometrial lining (dashed line)

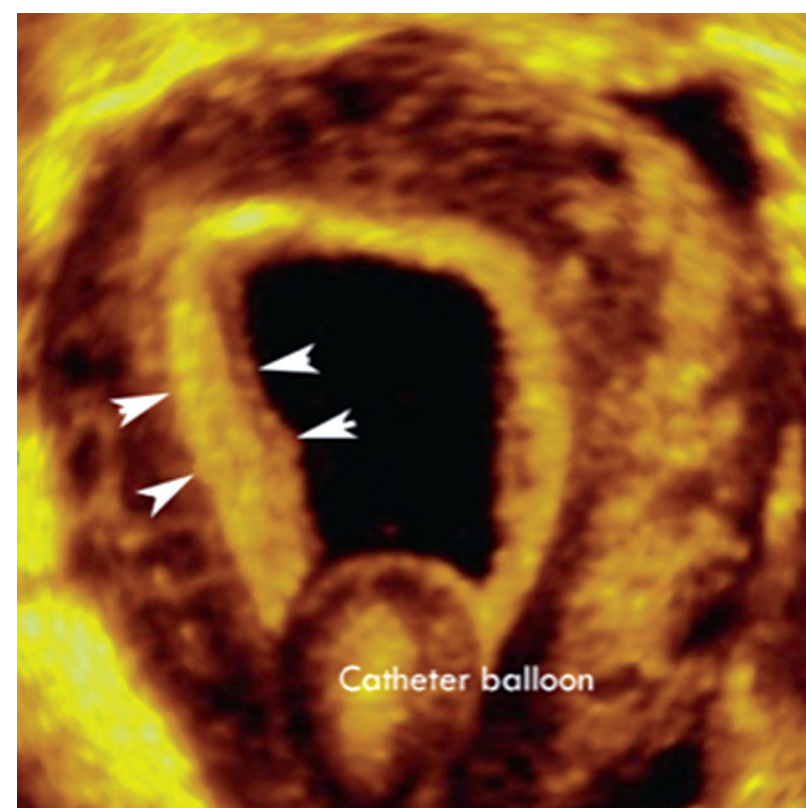

Figure 10. Endometrial hyperplasia in a 40-year-old woman with abnormal uterine bleeding. Three-dimensional saline infusion sonohysterography demonstrates the diffusely thickened endometrium (arrowheads). Pathological analysis revealed endometrial hyperplasia

sis and fibroids) and endometrial lesions. 3D SIS exhibits a very high detection rate; however, it cannot be used to exclude the presence of early or focal adenomyosis.

\section{Adhesions}

Adhesions are one of the causes of infertility, recurrent abortion, and reduced menstrual flow [7].

On sonohysterography, adhesions typically appear to be mobile, thin or thick echogenic bands that bridge a normally distensible endometrial cavity $[2,3,7,10]$. Synechiae can be mild, moderate, or severe according on whether adhesions involve one-fourth, one-half, or over three-fourths of the uterine cavity [7]. Furthermore, as the severity of adhesions progresses, the endometrial cavity may become difficult to distend during saline infusion, as in Asherman syndrome (Figure 9). Adhesions can also be associated with endometrial scars, which have a variable appearance ranging from small echogenic areas of focal endometrial thickening to areas of denuded endometrium [2].

3D SIS exhibits great value in the detection of adhesions and the assessment of the degree of endometrial cavity shape, dispensability, and volume.

\section{Endometrial hyperplasia}

Endometrial hyperplasia is a proliferative disorder of the endometrium, which usually results from unopposed estrogenic stimulation and can cause post-menopausal bleeding in $4-8 \%$ of cases [7].

On sonohysterography, endometrial hyperplasia is visualised as irregular, thickened, heterogeneous endometrium that is often diffusely distributed.

$3 \mathrm{D}$ rendering and volumetric studies offer better visualisation of diffuse or focal endometrial diseases and improved assessment of endometrial thickness (Figure 10).

\section{Congenital uterine anomalies}

Congenital uterine anomalies can arise from malformations at any step of the Müllerian developmental process and are present in $5.5 \%$ of the unselected population, $8 \%$ of infertile women, and $13.3 \%$ of women with histories of miscarriage [15]. Congenital uterine anomalies are commonly associated with infertility, recurrent miscarriage [15], and, in particular, an increased risk of first- and secondtrimester miscarriages and preterm delivery.

3D SIS appears promising for the diagnosis and classification of congenital uterine anomalies (Figure 11A). 3D SIS facilitates accurate evaluation of histological nature, intrauterine extent, and location of intrauterine abnormalities [17]. The ability to visualise both the uterine cavity and the external fundal uterine contour facilitates the diagnosis of uterine anomalies and enables differentiation between septate and bicornuate uteri [15]. One of the most useful scan planes obtained is the coronal view of the uterus, which is not usually obtainable on 2D US due to anatomical limitations (limited mobility of the vaginal probe within the confines of the vagina). These coronal views demonstrate the relationship between the endometrium and the myometrium at the uterine fundus, delineating the entire cervical canal and depicting the coronal angles [18].

3D Doppler ultrasound facilitates the visualisation of intraseptal vascularity and may help in distinguishing the avascular fibrous portion from the vascular septum of the myometrial component [18]. Less connective tissue in the septum may result in poor decidualisation and placenta- 


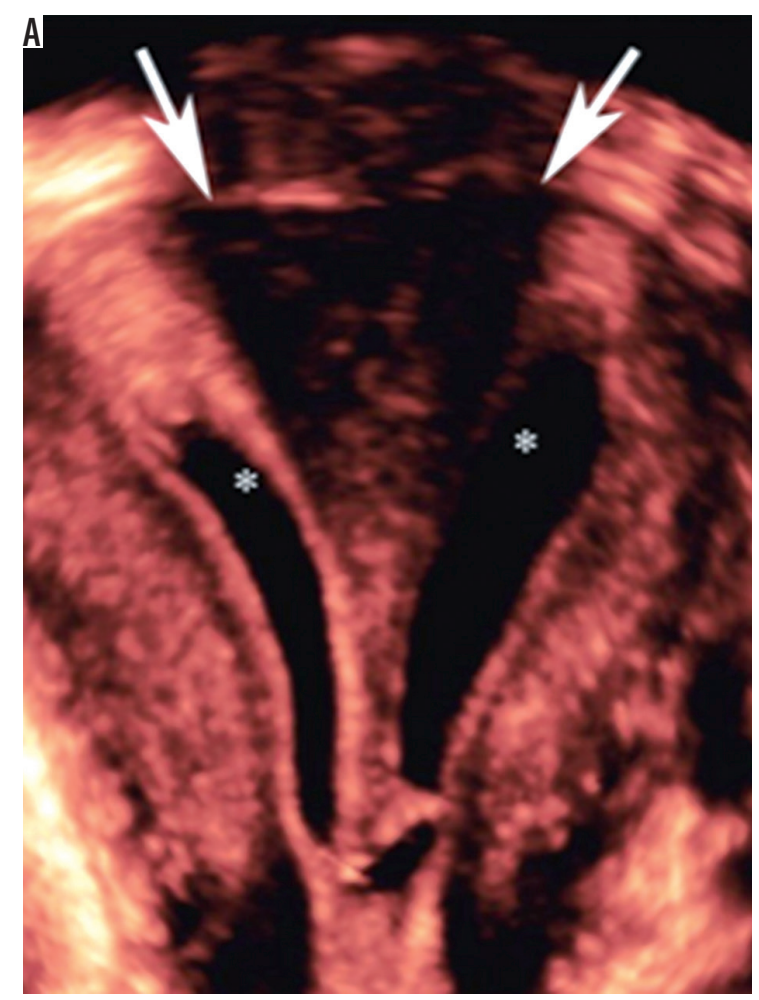

tion, whereas an increased amount of muscle tissue in the septum can cause miscarriage by the production of local uncoordinated myometrial contractility [18].

In addition to the diagnostic value of $3 \mathrm{D}$ sonohysterography in congenital uterine anomalies, such as unicornuate uterus and lesion inside the endometrial cavity (Figure 12A-B), this technique plays an important role in evaluating the endometrial cavity post-septal resection or metroplasty (Figure 11B) [19].

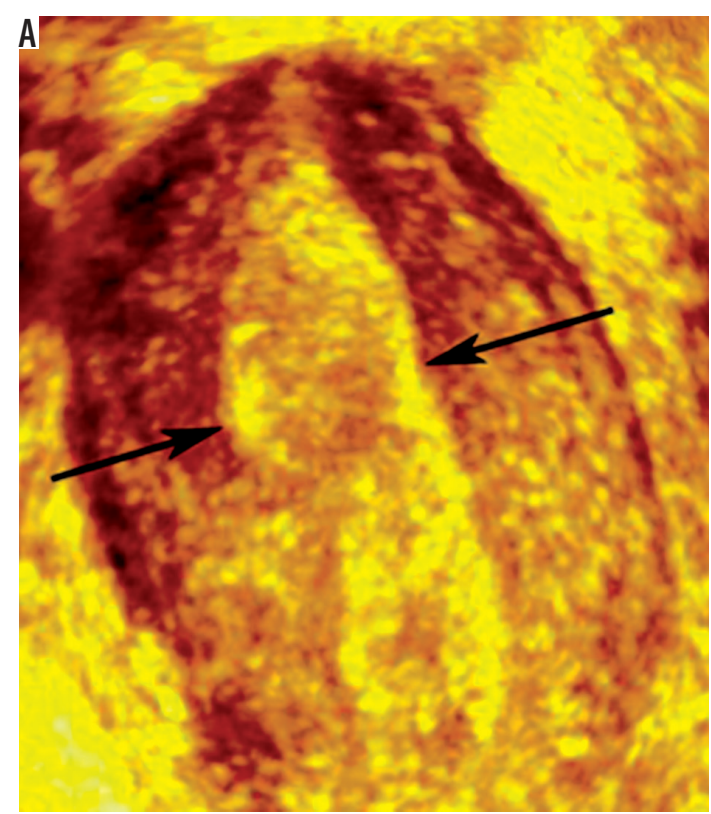

Figure 12. Unicornuate uterus in a 29-year-old woman with primary infertility. A) Three-dimensional (3D) ultrasound shows single uterine horn (arrows) with no divergence of the endometrium; B) 3D saline infusion sonohysterography demonstrates banana shaped uterus with single uterine horn

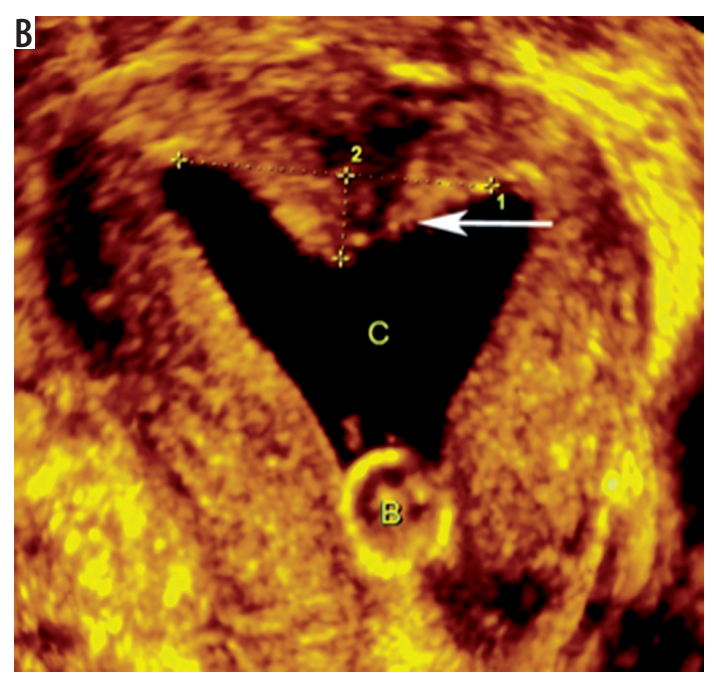

Figure 11. Septate uterus in a 21-year-old woman with a history of missed abortion. Pre-operative (A) Three-dimensional saline infusion sonohysterography (3D SIS) demonstrates two endometrial cavities (stars) communicating at the lower uterine segment, likely at the level of the internal os. The outer fundal contour (arrows) is maintained with a narrow angle $\left(<70^{\circ}\right)$ between the two cavities. B) 3D SIS after septal resection demonstrates improvement of the communicating uterine cavities $(C)$ with a residual septal length of $0.7 \mathrm{~cm}$ (arrow). The patient became pregnant and subsequently delivered

\section{Tubal patency}

Tubal factors are involved in $20-30 \%$ of infertile couples [20]. Therefore, the assessment of tubal patency is warranted as part of an infertility workup.

SIS is useful in the assessment of tubal patency because it is a fast, simple, minimally invasive, safe, and cheap technique without the risks of radiation [20].

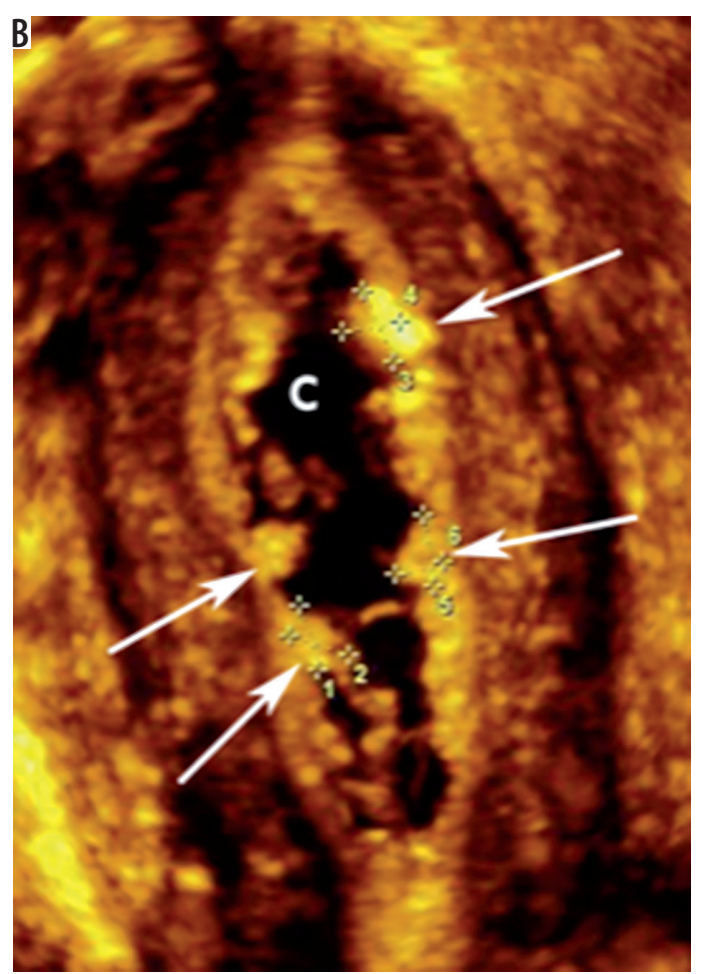



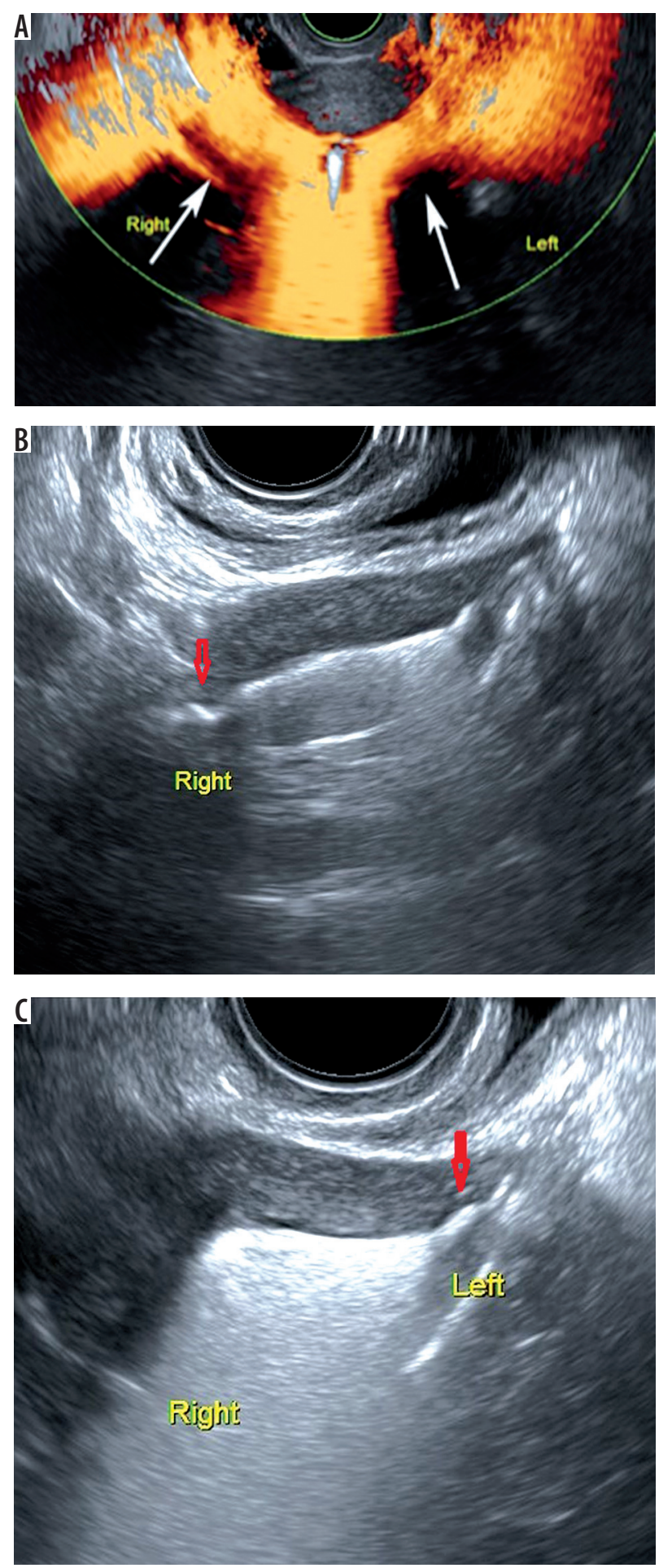

Figure 13. Patent fallopian tubes in a 26-year-old woman with primary infertility. A) saline infusion sonohysterography (SIS) with colour Doppler shows patent fallopian tubes (arrows). B-C) SIS after using foam gel contrast media (HyFoSy) transverse image at the proximal portion of the right and left tubes (arrows) reveals echogenic dispersion of foam in the fallopian tube and peritoneal cavity, which is compatible with patency of both tubes

Tubal patency may be indirectly assessed by colour Doppler during SIS by injecting air or saline (Figure 13A) and by measuring the amount of free fluid that accumulates during the procedure. A more quantitative evaluation requires the use of contrast material. The appearance

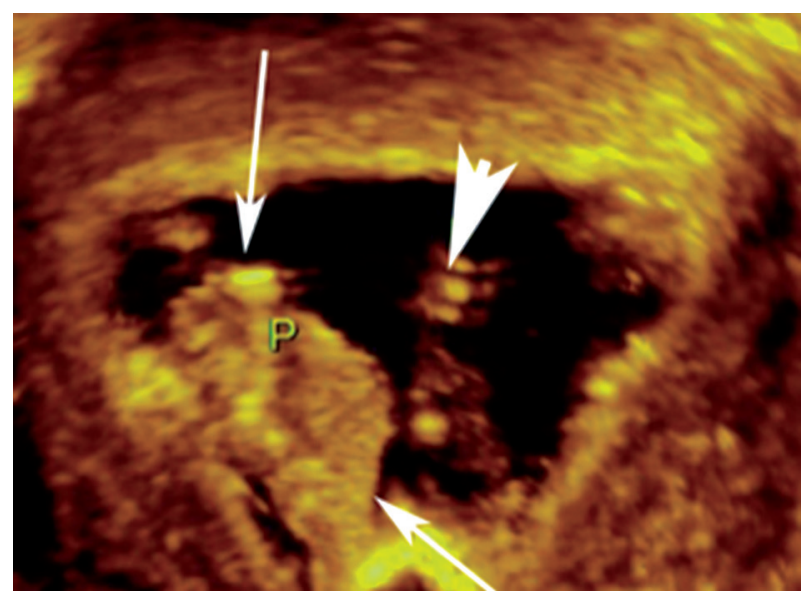

Figure 14. A polyp and synechiae in a 35-year-old woman with secondary infertility. Three-dimensional saline infusion sonohysterography demonstrates an endometrial polyp (arrows) at the lateral aspect of the uterine cavity. Additionally, an oblique fibrous band synechiae (arrowhead) is observed at the middle of the uterine cavity directed downwards

of fluid in the peritoneal cavity demonstrates at least unilateral tubal patency.

Some studies recommend the use of the foam gel HyFoSy (Figure 13B-C), which is feasible and accurate in the diagnosis of tubal patency, as in references [16,21].

\section{Mixed intrauterine pathologies}

Occasionally, a uterine pathology is associated with other pathologies; for example, polyps can co-occur with diffuse endometrial hyperplasia, fibroids, or synechiae. 3D SIS facilitates the accurate characterisation and localisation of different intrauterine pathologies (Figure 14).

\section{Summary}

There are few studies that compared 3D SIS to diagnostic hysteroscopy. In general, 3D SIS has high sensitivity and specificity in the diagnosis of various endometrial pathologies, particularly endometrial polyps and submucous fibroids. A study done by Khan et al. [5] reported detection with an accuracy of $97.5 \%$ for endometrial polyps, compared to $97.6 \%$ detection accuracy by diagnostic hysteroscopy. Also, they reported 3D SIS accuracy of $100 \%$ in the detection of submucous fibroids, compared to $91.6 \%$ detection by diagnostic hysteroscopy. Additionally, the diagnostic accuracy of 3D SIS in the evaluation of patients after hysteroscopy metroplasty is not significantly different from hysteroscopy, with a reported sensitivity of $97 \%$ and PPV of $100 \%$, compared to hysteroscopy [19].

In general SIS has higher specificity and sensitivity compared to TVUS. In a study done by Ryu et al. [22] in patients with abnormal uterine bleeding the reported sensitivity and specificity for TVUS are $79 \%$ and $45.8 \%$, and $95.1 \%$ and $83.3 \%$ for SIS, respectively. In another study by 
Erdem et al. [23] SIS had higher sensitivity and specificity for detection of endometrial polyps; they reported sensitivity and specificity for TVUS for endometrial polyps to be $70.5 \%$ and $90.2 \%$, respectively.

\section{Conclusions}

The 3D technique supplementary to the saline infusion sonohysterography procedure helps in the distinction between endometrial and myometrial lesions, and it facilitates delineation of uterine anatomy, resulting in more precise and accurate diagnosis.

\section{Conflict of interest}

The authors report no conflict of interest.

\section{References}

1. Allison SJ, Horrow MM, Kim HY, Lev-Toaff AS. Saline-infused sonohysterography: tips for achieving greater success. Radiographics 2011; 31: 1991-2004.

2. Davis PC, O’Neill MJ, Yoder IC, et al. Sonohysterographic findings of endometrial and subendometrial conditions. Radiographics 2002; 22: 803-816.

3. Weinraub Z, Maymon R, Shulman A, et al. Three-dimensional saline contrast hysterosonography and surface rendering of uterine cavity pathology. Ultrasound Obstet Gynecol 1996; 8: 277-282.

4. Sconfienza LM, Lacelli F, Caldiera V, et al. Three-dimensional sonohysterography forexamination of the uterine cavity in women with abnormal uterine bleeding: Preliminary findings. J Ultrasound 2010; 13: $16-21$.

5. Khan F, Jamaat S, Al-Jaroudi D. Saline infusion sonohysterography versus hysteroscopy for uterine cavity evaluation. Ann Saudi Med 2011; 31: 387-392.

6. Salim R, Lee C, Davies A, et al. A comparative study of three-dimensional saline infusion sonohysterography and diagnostic hysteroscopy for the classification of submucous fibroids. Hum Reprod 2005; 20: 253-257.

7. Zafarani F, Ahmadi F. Evaluation of intrauterine structural pathology by three-dimensional sonohysterography using an extended imaging method. Int J Fertil Steril 2013; 7: 1-6.

8. Bittencourt CA, Dos Santos Simões R, Bernardo WM, et al. Accuracy of saline contrast sonohysterography in detection of endometrial polyps and submucosal leiomyomas in women of reproductive age with abnormal uterine bleeding: systematic review and meta-analysis. Ultrasound Obstet Gynecol 2017; 50: 32-39.

9. Yung SS, Lai SF, Lam MT, et al. Randomized, controlled, double-blind trial of topical lidocaine gel and intrauterine lidocaine infusion for pain relief during saline contrast sonohysterography. Ultrasound Obstet Gynecol 2016; 47: 17-21.

10. Yang T, Pandya A, Marcal L, et al. Sonohysterography: Principles, technique and role in diagnosis of endometrial pathology. World J Radiol 2013; 5: 81-87.

11. Salim S, Won H, Nesbitt-Hawes E, et al. Diagnosis and management of endometrial polyps: a critical review of the literature. J Minim Invasive Gynecol 2011; 18: 569-581.

12. Cullinan JA, Fleischer AC, Kepple DM, Arnold AL. Sonohysterography: a technique for endometrial evaluation. Radiographics 1995 15: 501-514; discussion 515-516.
13. Alatas C, Aksoy E, Akarsu C, et al. Evaluation of intrauterine abnormalities in infertile patients by sonohysterography. Hum Reprod 1997; 12: 487-490.

14. Verma SK, Lev-Toaff AS, Baltarowich OH, et al. Adenomyosis: sonohysterography with MRI correlation. Am J Roentgenol 2009; 192: 1112-1116.

15. Ludwin A, Pityński K, Ludwin I, et al. Two- and three-dimensional ultrasonography and sonohysterography versus hysteroscopy with laparoscopy in the differential diagnosis of septate, bicornuate, and arcuate uteri. J Minim Invasive Gynecol 2013; 20: 90-99.

16. Lindheim SR, Adsuar N, Kushner DM, et al. Sonohysterography: a valuable tool in evaluating the female pelvis. Obstet Gynecol Surv 2003; 58: 770-784.

17. de Kroon CD, Louwé LA, Trimbos JB, Jansen FW. The Clinical Value of 3-Dimensional Saline Infusion Sonography in Addition to 2-Dimensional Saline Infusion Sonography in Women with Abnormal Uterine Bleeding. J Ultrasound Med 2004; 23: 1433-1440.

18. Kupesic S. Clinical implications of sonographic detection of uterine anomalies for reproductive outcome. Ultrasound Obstet Gynecol 2001; 18: 387-400.

19. Ludwin A, Ludwin I, Kudla M, et al. Diagnostic accuracy of three-dimensional sonohysterography compared with office hysteroscopy and its interrater/intrarater agreement in uterine cavity assessment after hysteroscopic metroplasty. Fertil Steril 2014; 101: 1392-1399.

20. Malek-Mellouli M, Gharbi H, Reziga H. The value of sonohysterography in the diagnosis of tubal patency among infertile patients. Tunis Med 2013; 91: 387-390.

21. Van Schoubroeck D, Van den Bosch T, Meuleman C, et al. The use of a new gel foam for the evaluation of tubal patency. Gynecol Obstet Invest 2013; 75: 152-156.

22. Ryu JA, Kim B, Lee J, et al. Comparison of transvaginal ultrasonography with hysterosonography as a screening method in patients with abnormal uterine bleeding. Korean J Radiol 2004; 5: 39-46.

23. Erdem M, Bilgin U, Bozkurt N, Erdem A. Comparison of transvaginal ultrasonography and saline infusion sonohysterography in evaluating the endometrial cavity in pre- and postmenopausal women with abnormal uterine bleeding. Menopause 2007; 14: 846-852. 